\title{
Artéria renal dupla originando da aorta em cão: relato de caso
}

\section{Double renal artery originating from aorta in dog: case report}

\author{
Luciano da Silva Alonso $^{1 *}$; Marcelo Abidu-Figueiredo ${ }^{2}$
}

\section{Resumo}

A presença de artéria renal múltipla em cães ocorre geralmente do lado esquerdo, em uma freqüência de 12 a $15 \%$. O avanço de técnicas cirúrgicas no trato urogenital e a utilização de modelos para transplantes renais tornam importante o conhecimento das variações possíveis no que se refere à vascularização dos rins. Cursos de anatomia veterinária e anatomia animal direcionados para estudantes de cursos de graduação nas áreas de ciências agrárias e biológicas, utilizam material anatômico proveniente de animais domésticos em volume considerável. A utilização do acervo dos laboratórios de anatomia para estudos de freqüência de variações anatômicas constitui meio de contribuição junto às áreas aplicadas, tais como a cirurgia e a clínica. O objetivo deste relato é apresentar um caso de artéria renal esquerda dupla, com origem na porção ventral da aorta, em cadáver de cão sem raça definida, com cerca de dois meses de idade, preservado em solução de formol a 10\% e com injeção vascular de látex corado.

Palavras-chave: Cão, anatomia, artéria renal dupla

\begin{abstract}
The presence of the multiple renal artery in dogs, supplying the left kidney, has been reported in $12-15 \%$. New surgery technics in the urogenital tract and the utilization of model for renal transplantation are of clinical significance when considering surgery of that kidney. Courses of anatomy directed veterinary medicine and animal anatomy for students of courses of graduation in the areas of agrarian and biological sciences, use anatomical material proceeding from domestic animals in considerable volume. The use of the material of the laboratories of anatomy for studies of frequency of anatomical variations constitutes way of together contribution to the applied areas, such as the surgery and the clinic. The objective of this report is to presentation of the left multiple renal artery case, originating from the ventral portion of the aorta, in female dog cadaver, with two months, formalin-preserved at $10 \%$ and with latex colored vascular injected.
\end{abstract}

Key words: Dog, anatomy, double renal artery

UFRRJ - IB/DBA/Anatomia Animal. Brasil - Seropédica - RJ cep: 23890-000 E-mail: alonsols@ufrrj.br

2 Instituto de Biologia/Departamento de Biologia Animal/Área de Anatomia Animal.

* Autor para correspondência 


\section{Introdução}

O conhecimento das variações da anatomia vascular renal possui grande importância na exploração semiótica dos rins e outras condições, tais como tratamento do trauma renal, transplante renal, hipertensão renovascular, embolização da artéria renal, angioplastia ou reconstrução vascular para lesões congênitas ou adquiridas, cirurgia para aneurisma da aorta abdominal e cirurgia renal conservadora ou radical (SAMPAIO, ARAGÃO, 1990).

Nos mamíferos as artérias renais direita e esquerda fornecem suprimento sangüíneo para os rins (GETTY， 1986; NICKEL; SCHUMMER; SEIFERLE, 1979, 1981), sendo a origem destes vasos a cada lado da porção abdominal da aorta. (JAIN; SINGH, 1987; NICKEL; SCHUMMER; SEIFERLE, 1981). Cada artéria renal dá origem aos ramos dorsal e ventral, antes de entrar no hilo renal. Os ramos dorsal e ventral respectivamente, se dividem nas artérias inter-lobares, arquedas e interlobulares (AKSOY; OZUDOGRU, 2003).

Embora trabalhos com enfoque na vascularização do retroperitônio e do pedículo renal tenham sido realizados ao longo dos últimos anos, em geral as descrições encontradas na literatura são genéricas, inclusive no que se refere à vascularização dos órgãos urogenitais e adjacentes, com pouca ênfase a implicações cirúrgicas e radiológicas (SAMPAIO; PASSOS, 1992).

Recentes avanços e refinamentos nas técnicas utilizadas em cirurgia urológica e no diagnóstico por imagem motivaram o desenvolvimento de estudos sobre vascularização dos rins, sendo imprescindível o conhecimento anatômico para realizar manobras intervencionistas de forma segura e eficaz (FARINON et al. 1984; SAMPAIO; PASSOS, 1992). Portanto, o conhecimento das variações anatômicas da artéria renal é importante tanto para abordagens cirúrgicas em animais de experimentação utilizados em pesquisa e treinamento cirúrgico, como também para evitar complicações desnecessárias em cirurgias.

Ao avaliar a ocorrência de variação da artéria renal na espécie humana, Sampaio e Passos (1992) observaram a existência de vários arranjos, com duplicação ou triplicação daquele vaso. Os mesmos autores citaram a ocorrência de ramos arteriais suprindo tanto o pólo superior do rim quanto o pólo inferior. Conforme estes autores, a nomenclatura utilizada para a denominação de tais variações apresenta inconsistência, pois são utilizados termos como "extra", "aberrante" ou "acessório", inadequados neste contexto por tratar-se de segmentos terminais normais, sem anastomose entre eles. Estes vasos correspondem a ramos segmentares adicionais para o suprimento dos rins. O termo "supernumerário" também deve ser evitado porquê pode sugerir que tais vasos são supérfluos. A ligadura de qualquer artéria direcionada ao parênquima renal produzirá danos ao mesmo, fortalecendo a tese dos autores de que tais termos não são adequados. Visando contribuir para a adequada denominação destas formas variantes, os autores sugerem a utilização do termo "artérias renais múltiplas", entendendo que as mesmas são de máxima importância prática.

Ainda fazendo referência à espécie humana, a presença de artérias renais múltiplas aumentam a complexidade do transplante renal (FOX; YALIN, 1979) assim como há maior correlação com patologias renais em comparação aos órgãos supridos por artéria renal única (SAMPAIO; PASSOS, 1992).

Em trabalho sobre freqüência de duplicação da artéria renal foram encontrados 29 casos de duplicação da artéria renal esquerda em 117 cães examinados por dissecção (CRHISTENSEN, 1952). Em outro estudo, realizado com rins de 500 cães (REIS; TEPE, 1956), foi encontrado, em 99,4 \%, a artéria renal direita se apresentando de forma única; em $12,8 \%$ dos casos a artéria renal esquerda foi descrita como dupla; e em $0,4 \%$ observou-se artéria renal esquerda de forma tripla. Getty (1986) descreve a vascularização do rim de cães, citando apenas que é possível que a artéria renal seja dupla, especialmente no rim esquerdo. Outros autores descrevem as ramificações das artérias renais de cães, mas não comentam sobre as possíveis variações que possam ocorrer (NICKEL; SCHUMMER; SEIFERLE, 1979; EVANS; DE LAHUNTA, 1994). 
O objetivo deste artigo é relatar um caso de duplicação da artéria renal esquerda em cadáver de cão e enfatizar a importância do conhecimento da ocorrência de variações anatômicas na prática clínicocirúrgica.

\section{Relato do Caso}

Relata-se o caso de cão do sexo feminino, sem raça definida, com comprimento rostro-sacral de 30,5 $\mathrm{cm}$, com aproximadamente dois meses de idade e pesando 1,5 kg, o qual foi doado à Área de Anatomia Animal da Universidade Federal Rural do Rio de Janeiro após óbito por causas não relacionadas ao trato urogenital. Após o posicionamento do animal em decúbito lateral realizou-se a abertura do tórax, dissecação e canulação da porção torácica da aorta. Através da mesma foi feita a fixação com solução de formaldeído a $10 \%$. Em seguida foram feitas repleções vasculares com solução de Petrolátex S 65 corado em vermelho. No ato da dissecação observou-se a presença de duplicação da artéria renal esquerda (Figura 1). O vaso mais cranial apresentouse ligeiramente mais delgado que o vaso caudal, à inspeção visual. Os dois vasos originaram-se da porção ventral da aorta, com distância de $0,2 \mathrm{~cm}$ entre as origens. O comprimento dos ramos cranial e caudal foi, respectivamente, de 2,2 cm e 2,1 cm. Após cursar trajeto retilíneo e convergente em direção ao hilo renal, penetraram no rim após bifurcação. As demais estruturas apresentaram-se com morfologia dentro da normalidade. A artéria renal direita originou-se da porção lateral da aorta, sendo única e bifurcando-se no hilo renal, seguindo o padrão mais usual para este vaso. Os outros órgãos abdominais foram avaliados e apresentaram-se com aspecto macroscópico normal e sem variações quanto ao arranjo vascular. A figura 2 apresenta a esquematização da duplicidade da artéria renal.

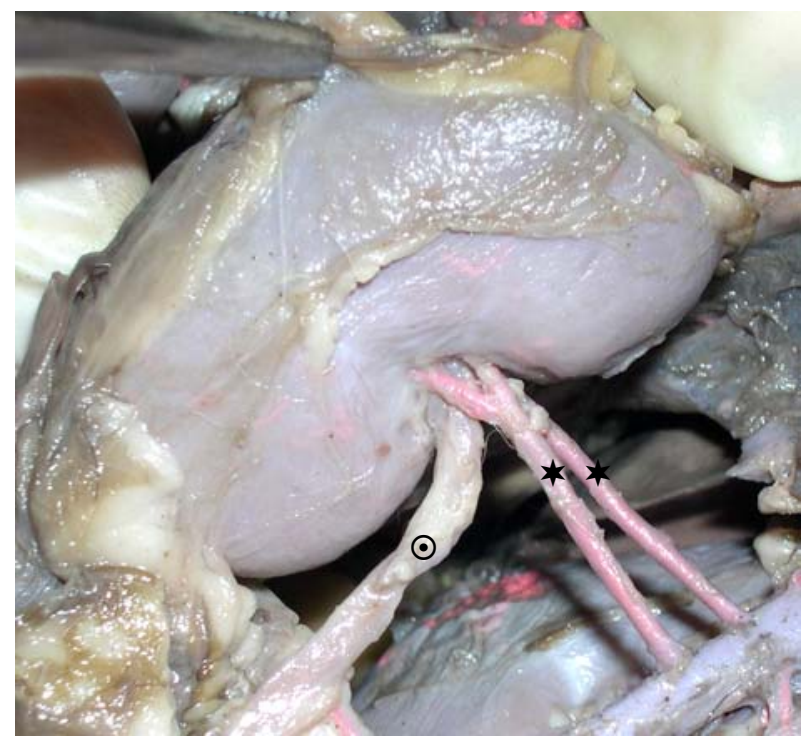

Figura 1. Fotomacrografia do rim esquerdo com duplicação da artéria renal.

$*=$ artérias renais. $\odot=$ ureter

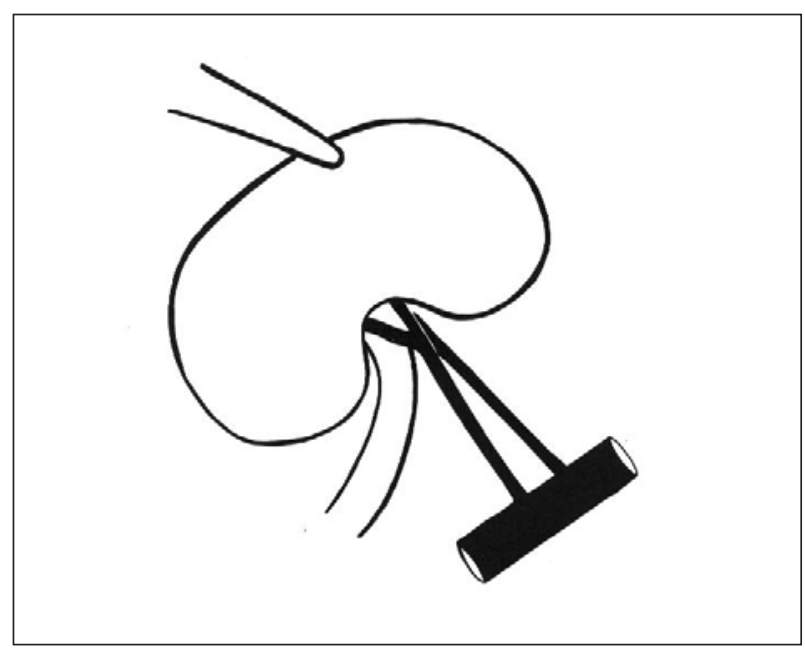

Figura 2. Esquema demonstrando o rim esquerdo com duplicação da artéria renal.

\section{Discussão}

As artérias renais esquerdas múltiplas se originaram da porção ventral da aorta, contrastando com as informações de outros autores que mencionam origem lateral destes vasos (NICKEL; SCHUMMER; SEIFERLE, 1979, 1981; JAIN; SINGH, 1987; AKSOY; OZUDOGRU, 2003). 
Não foram encontradas referências sobre possíveis variações de número dos componentes do pedículo renal em cães (NICKEL; SCHUMMER; SEIFERLE, 1979, 1981; EVANS; DE LAHUNTA; 1994; KONIG; MAIERL; LIEBICH, 2004). Getty (1986) ao descrever a vascularização do rim de cães, cita apenas que é possível que a artéria renal seja dupla, especialmente no rim esquerdo.

Relatos de variação numérica da artéria renal em cães foram realizados na década de 1950 (CHRISTENSEN, 1952; REIS; TEPE, 1956). Desde então pouca atenção tem sido dada ao estudo das variações vasculares nos rins de cães, sobretudo correlacionadas ao desenvolvimento de novas técnicas intervencionistas.

$\mathrm{Na}$ literatura cirúrgica do trato urogenital, encontram-se citações sobre a presença de artérias renais múltiplas suprindo o rim esquerdo (FOSSUM, 2002). A ocorrência de artérias renais múltiplas no rim esquerdo foi descrita na freqüência de 12 a $15 \%$ dos cães (LANZ; WALDRON, 2000), sendo necessário verificar possíveis artérias múltiplas suprindo o rim esquerdo por ocasião de cirurgias no órgão, segundo os autores.

O setor de Anatomia Animal da Universidade Federal Rural do Rio de Janeiro utiliza cadáveres de cães doados pelo Hospital Veterinário desta Universidade, como rotina nas disciplinas destinadas ao Curso de Medicina Veterinária. Durante vários anos de atividades de dissecação ininterruptas não havia sido observado, por este grupo, variação na vascularização dos rins de cães, até o presente caso.

A divulgação dos achados das variações anatômicas é importante para a prática médico veterinária e deve ser realizada como forma de conscientização dos profissionais, contribuindo para o sucesso e aperfeiçoamento de diferentes protocolos clínico-cirúrgicos.

\section{Referências}

AKSOY, G.; OZUDOGRU,Z. A macroscopical investigation on the intrarenal segmentation of the renal arteries in the
Van cat (in Turkish). Journal of the Faculty Veterinary Medicine, Kars, v.9, p.9-1, 2003.

CHRISTENSEN, G. C. Circulation of blood through the canine kidney. American Journal of Veterinary Research, Chicago, v.13, p.236-245, 1952.

EVANS, H. E.; DE LAHUNTA, A. Guia para a dissecção do cão. Rio de Janeiro: Guanabara Koogan, 1994.

FARINON, A. M.; LAMPUGNANI, R.; ZANNONI, M.; DELFRATE, R.; FREDDI, M.;: In tema de Anatomia Chirurgica dei Vasi Arteriosi Addominali Nei Piu Comuni Animali da Esperimento. Chir. e Pat. Sper., 32 (1)17-27. 1984.

FOSSUM, W. T. Cirurgia dos rins e dos ureteres. In: Roca, 2002. Cirurgia de pequenos animais. 2.ed. São Paulo:

FOX, M.; YALIN, R. Renal transplantation with multiple arteries. British Journal of Urology, Edinburgh, v.51, p.333-336, 1979.

GETTY, R. Anatomia dos animais domésticos. 5.ed. Rio de Janeiro: Guanabara Koogan, 1986.

JAIN R. K.; SINGH, Y. Vascularization of kidneys in bovine calves. Indian Veterinary Journal, Madras, v.64, p.10591062, 1987.

KÖNIG, H. E.; MAIERL, J.; LIEBICH, H. G. Órgãos urinários (organa urinaria). In: KONIG, H. E.; LIEBICH, H. G. Anatomia dos animais domésticos: texto e atlas colorido. Porto Alegre: Artmed, 2004. p.103-118.

LANZ, O. I.; WALDRON, D. R. Renal and ureteral surgery in dogs. Clinical Techniques in Small Practice. Philadelphia, v.15, p.1-10, 2000.

NICKEL, R.; SCHUMMER, A.; SEIFERLE, E. The viscera of domestic mammals. 2.ed. Berlin: Verlag Paul Parey,1979. The anatomy of the domestic animals. Berlin: Verlag Paul Parey, 1981.

REIS, R. H.; TEPE P. Variations in the pattern ofrenal vessels and their relation to the type posterior vena cava in the dog (Canis Familiaris). American Journal of Anatomy, Philadelphia, v.99, p.1-15, 1956.

SAMPAIO, F.; ARAGÃO, A. H. B. M. Anatomical relationship between the intrarenal arteries and the kidney collecting system. Journal Of Urology, Baltimore, v.143, p.679-681, 1990.

SAMPAIO, F. J. B.; PASSOS, M. A. R. F. Renal arteries: anatomic study for surgical and radiological practice. Surgical and Radiologic Anatomy, Paris, v.14, p.113-117, 1992. 